\title{
I I 0 I Comparison of self-gating synchronization algorithms for cardiac cine MRI
}

\author{
Grace M Nijm*1, Alan V Sahakian ${ }^{1}$, Steven Swiryn ${ }^{1,2}$ and Andrew C Larson ${ }^{1}$
}

Address: ${ }^{1}$ Northwestern University, Evanston, IL, USA and ${ }^{2}$ HeartCare Midwest, Peoria, IL, USA

* Corresponding author

from I th Annual SCMR Scientific Sessions

Los Angeles, CA, USA. I-3 February 2008

Published: 22 October 2008

Journal of Cardiovascular Magnetic Resonance 2008, I0(Suppl I):A226 doi:10.1 I86/I532-429X-10-SI-A226

This abstract is available from: http://jcmr-online.com/content/I0/SI/A226

(c) 2008 Nijm et al; licensee BioMed Central Ltd.

Interference from gradient switching, radiofrequency pulses, and the magnetohydrodynamic effect distort ECG signals typically used for gating in cardiac MRI, potentially resulting in imprecise triggering. Furthermore, the use of a lead system during a MRI scan requires additional equipment and patient setup time. Self-gating (SG) is an alternative technique which directly utilizes MRI signals for cardiac gating; consequently, synchronization is related to the intrinsic motion of the heart instead of electrical activity. Previous work in SG used a simple first difference peak detection scheme to identify trigger times. We hypothesized that the SG trigger detection accuracy can be improved by using more advanced signal processing techniques.

Fourteen healthy volunteers underwent cardiac MRI using a 1.5 Tesla MRI scanner. SG signals were obtained for two chamber, three chamber, four chamber, and short axis scans. Three peak detection schemes were implemented for comparison with the ECG triggers. The first method involved first differences (FD). The first derivative of the SG signal was computed and positive-to-negative sign change locations were identified; thresholding was then applied to identify local maxima of the original signal. The second method involved template matching (TM). This was accomplished by generating the median template and computing the cross-correlation signal between the median template and the original signal. The peaks of the cross-correlation signal were detected and identified as the triggers. The third method involved polynomial fitting (PF) with a cubic. The metric to assess SG trigger time accuracy was root-mean-squared (RMS) error, defined as variability relative to mean difference between SG and ECG trigger times. Variability relative to this mean was chosen to account for constant phase differences between the triggering schemes. P-values were computed to determine if more advanced trigger detection methods (TM and cubic PF) provided a statistically significant improvement over the original method (FD). Images were reconstructed based upon each trigger detection method; mean

Table I: Mean \pm standard deviation of the RMS errors; p-values shown in parentheses

\begin{tabular}{|c|c|c|c|}
\hline & First Differences & Template Matching & Cubic Polynomial Fitting \\
\hline Two chamber & $24.86 \pm 11.47 \mathrm{~ms}$ & $\begin{array}{l}17.44 \pm 5.72 \mathrm{~ms} \\
(p=0.006)\end{array}$ & $\begin{array}{l}15.54 \pm 7.27 \mathrm{~ms} \\
(p=0.007)\end{array}$ \\
\hline Three chamber & $17.28 \pm 10.88 \mathrm{~ms}$ & $\begin{array}{l}12.75 \pm 7.51 \mathrm{~ms} \\
(p=0.015)\end{array}$ & $\begin{array}{l}10.20 \pm 4.60 \mathrm{~ms} \\
(p=0.016)\end{array}$ \\
\hline Four chamber & $20.58 \pm 7.79 \mathrm{~ms}$ & $\begin{array}{l}14.88 \pm 5.04 \\
\mathrm{~ms}(\mathrm{p}=0.026)\end{array}$ & $\begin{array}{l}12.97 \pm 6.00 \\
\mathrm{~ms}(\mathrm{p}=0.026)\end{array}$ \\
\hline Short axis & $10.55 \pm 4.84 \mathrm{~ms}$ & $\begin{array}{l}8.96 \pm 3.44 \mathrm{~ms} \\
(p=0.344)\end{array}$ & $\begin{array}{l}7.83 \pm 3.64 \mathrm{~ms} \\
(p=0.103)\end{array}$ \\
\hline
\end{tabular}


Table 2: MSE, PSNR and p-values for image comparison

\begin{tabular}{|c|c|c|c|c|c|c|}
\hline & MSE & & & PSNR (dB) & & \\
\hline & First Differences & Cubic Polynomial Fitting & P-value & First Differences & Cubic Polynomial Fitting & P-value \\
\hline Two Chamber & $11.3 \pm 8.2$ & $9.1 \pm 5.8$ & 0.0317 & $38.9 \pm 3.0$ & $39.8 \pm 2.8$ & 0.0006 \\
\hline Three Chamber & $18.4 \pm 12.8$ & $15.2 \pm 12.1$ & 0.0002 & $36.9 \pm 3.6$ & $37.8 \pm 3.5$ & 0.0014 \\
\hline Four Chamber & $16.2 \pm 8.8$ & $12.0 \pm 9.4$ & 0.0188 & $37.1 \pm 2.7$ & $38.7 \pm 3.2$ & 0.0111 \\
\hline Short Axis & $9.7 \pm 5.4$ & $8.4 \pm 4.8$ & 0.0059 & $39.7 \pm 3.0$ & $40.0 \pm 3.0$ & 0.0418 \\
\hline
\end{tabular}

squared error (MSE) and peak signal-to-noise ratio (PSNR) of the images were calculated to compare image quality.

The average RMS errors were lower for both the TM and cubic PF than for the FD methods for all scan types. Mean \pm standard deviation of the RMS errors and p-values are shown in Table 1. The p-values indicate that the lower RMS errors for both TM and cubic PF were statistically significant for all scan orientations except for short axis. MSE and PSNR were calculated for images generated by FD and cubic PF trigger methods relative to ECG-gated images. Since cubic PF performed better in trigger detection than $\mathrm{TM}$, it was chosen for comparison of image quality with the original FD method. These data as well as p-values are shown in Table 2. The p-values indicate that the lower MSE and higher PSNR values for cubic PF were statistically significant for all scan orientations.

In conclusion, since SG signals are highly dependent upon in-plane and through-plane blood flow patterns, two, three, and four chamber orientations may result in more complex signal morphologies, requiring more advanced signal processing techniques for accurate trigger detection. Consequently, at these scan orientations, both TM and cubic PF methods provided improved SG trigger detection in comparison with FD. Furthermore, comparison of the images indicated that improvements in trigger detection were reflected in improvements in image quality.

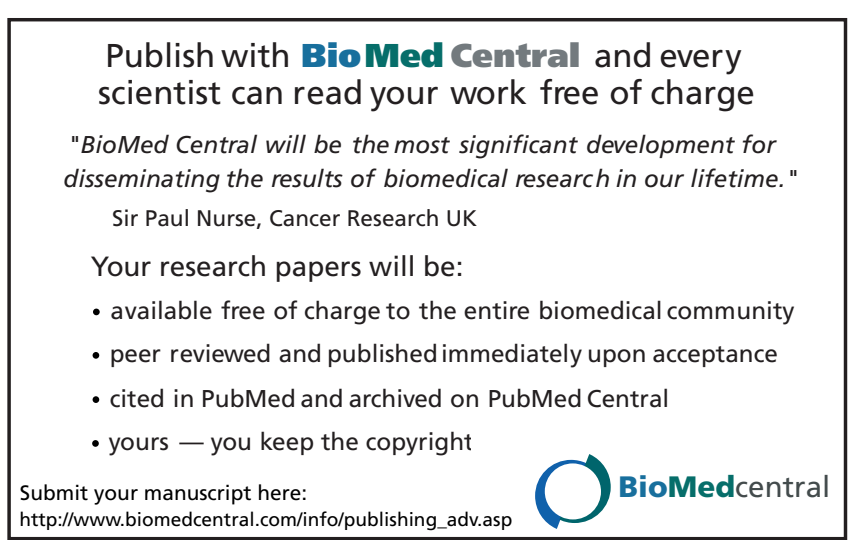

Page 2 of 2

(page number not for citation purposes) 